ARTICLE

\title{
Eosinophil function in adipose tissue is regulated by Krüppel-like factor 3 (KLF3)
}

\author{
Alexander J. Knights (1) ${ }^{1}$, Emily J. Vohralik ${ }^{1}$, Peter J. Houweling ${ }^{2,3}$, Elizabeth S. Stout ${ }^{1}$, Laura J. Norton ${ }^{1}$, \\ Stephanie J. Alexopoulos ${ }^{1}$, Jinfen. J. Yik ${ }^{1}$, Hanapi Mat Jusoh ${ }^{4}$, Ellen M. Olzomer ${ }^{1}$, Kim S. Bell-Anderson ${ }^{4}$, \\ Kathryn N. North ${ }^{2,3}$, Kyle L. Hoehn (1) ${ }^{1}$, Merlin Crossley (D) ${ }^{1} \&$ Kate G. R. Quinlan (D) ${ }^{1 \times}$
}

\begin{abstract}
The conversion of white adipocytes to thermogenic beige adipocytes represents a potential mechanism to treat obesity and related metabolic disorders. However, the mechanisms involved in converting white to beige adipose tissue remain incompletely understood. Here we show profound beiging in a genetic mouse model lacking the transcriptional repressor Krüppel-like factor 3 (KLF3). Bone marrow transplants from these animals confer the beige phenotype on wild type recipients. Analysis of the cellular and molecular changes reveal an accumulation of eosinophils in adipose tissue. We examine the transcriptomic profile of adipose-resident eosinophils and posit that KLF3 regulates adipose tissue function via transcriptional control of secreted molecules linked to beiging. Furthermore, we provide evidence that eosinophils may directly act on adipocytes to drive beiging and highlight the critical role of these little-understood immune cells in thermogenesis.
\end{abstract}

\footnotetext{
${ }^{1}$ School of Biotechnology and Biomolecular Sciences, UNSW Sydney, Sydney, NSW 2052, Australia. ${ }^{2}$ Murdoch Children's Research Institute, The Royal Children's Hospital, Melbourne, VIC 3052, Australia. ${ }^{3}$ Department of Paediatrics, University of Melbourne, Melbourne, VIC 3052, Australia. ${ }^{4}$ Charles Perkins Centre, School of Life and Environmental Sciences, University of Sydney, Sydney, NSW 2006, Australia. ${ }_{\text {email: kate.quinlan@unsw.edu.au }}$
} 
W hite adipose tissue (AT), typically regarded as an energy storage site, can acquire the thermogenic properties of brown AT to become 'beige' and drive energy expenditure ${ }^{1,2}$. The discovery that AT can be activated in this way may be important in combatting obesity and associated cardiometabolic disorders.

It is now apparent that resident immune cells, particularly mediators of type 2 immunity, are involved in beige AT activation and energy expenditure. Eosinophils have been implicated in beiging ${ }^{3-5}$ but the underlying cellular and molecular mechanisms orchestrating their contributions remain incompletely understood. Obese mice have fewer eosinophils in their AT than lean counterparts, and the $\Delta$ dblGATA transgenic mouse, which lacks eosinophils altogether, displays exaggerated weight gain on a high-calorie $\operatorname{diet}^{4}$. Using the $\Delta$ dblGATA model, it has also been proposed that AT eosinophils facilitate adipocyte maturation to ameliorate diabetic complications of diet-induced obesity ${ }^{6}$. Conversely, mice genetically engineered to overexpress interleukin 5 (IL-5) have supraphysiological levels of eosinophils and are protected from diet-induced obesity ${ }^{4}$. However, a recent study reported that artificially increasing eosinophils in AT with recombinant IL-5 did not confer the expected metabolic benefits, leading the authors to suggest that the functional activities of eosinophils may be more crucial than their abundance ${ }^{7}$. Furthermore, the principal contribution of macrophages to AT energy expenditure-their production of catecholamines ${ }^{8}$-is currently under debate, with several groups finding that rather than synthesising catecholamines, macrophages are instead responsible for catecholamine uptake and degradation ${ }^{9-11}$. Thus, interest in the key cellular and molecular drivers of beiging remains intense.

We have previously shown that mice lacking the transcriptional repressor Krüppel-like factor 3 (KLF3) have reduced adiposity and are protected from diet-induced obesity ${ }^{12,13}$. Here we report enhanced beige AT activation in $\mathrm{Klf3}^{-/-}$mice and that bone marrow $(\mathrm{BM})$ transplants from these mice confer the lean, beige phenotype on recipients. In the absence of KLF3, ATresident eosinophils are more abundant and exhibit significant deregulation of important secreted molecules, including meteorin-like and IL-33, both of which influence beiging $5,14-16$. We also report that co-culture of eosinophils with primary adipocytes increases thermogenic gene expression. These findings identify KLF3 as an important regulator of AT eosinophil gene expression and function, advancing our understanding of how these little-understood immune cells may lead to improved strategies for therapeutically driving energy expenditure.

\section{Results}

Reduced adiposity and enhanced beiging in $K l f 3^{-/-}$mice. Disruption of the gene encoding the transcriptional repressor KLF3 results in mice that are smaller than their wild type (WT) littermates with less white $\mathrm{AT}^{13}$ (Supplementary Fig. 1a, b), and confers protection from diet-induced obesity ${ }^{12}$. Body mass composition analysis of chow-fed WT and $\mathrm{Klf3}^{-/-}$animals housed at room temperature $\left(22^{\circ} \mathrm{C}\right)$ showed that $\mathrm{Klf3} 3^{-/-}$mice exhibit reduced total fat mass compared to WT littermates (Fig. 1a and Supplementary Fig. 1a), in addition to differences in lean body mass, which constitute their reduced body weight (Supplementary Fig. 1b). This is reflected in the reduced size of white AT depots in $\mathrm{Klf3}^{-/-}$mice seen previously ${ }^{12}$ (Fig. $1 \mathrm{~b}$ and Supplementary Fig. 1c). Visual examination of subcutaneous (subcut) AT depots, the depots most prone to beiging ${ }^{17}$, revealed a browner complexion and smaller size in $\mathrm{Klf3}^{-1-}$ mice (Fig. 1c). Furthermore, H\&E staining revealed that in the absence of KLF3, adipose cellular architecture is notably altered, with enrichment of multilocular adipocytes evident that was not seen in the subcut AT of WT mice (Supplementary Fig. 2a, b). These observations also confirm the previous finding that $\mathrm{Klf}^{-/-}$mice have smallersized adipocytes ${ }^{12,13}$. Given that thermogenic energy expenditure via activation of beige AT may influence adiposity ${ }^{18-20}$, we examined the expression of archetypal thermogenic genes. We observed upregulation of numerous thermogenic genes in the subcut AT of $\mathrm{Klf3}^{-/-}$mice - most notably Ucp 1, Cpt1b, Fatp1 and the beige-specific marker ${ }^{21}$ Tbx1 (Fig. 1d). We next investigated the levels of mitochondrial proteins by Western blotting of whole-cell extracts (WCE) from WT and $\mathrm{Klf}^{-/-}$subcut AT. Uncoupling protein 1 (UCP1) protein levels were higher in the subcut AT of $\mathrm{Klf3}^{-/-}$mice (Fig. 1e), as were mitochondrial oxidative phosphorylation (oxphos) complexes I-V (Fig. 1f). We also observed increased levels of the mitochondrial outer membrane protein voltage-dependent anion channel (VDAC) in $\mathrm{Klf}^{-/-}$subcut AT (Fig. 1g), suggesting higher mitochondrial number. Levels of multiple thermogenic genes were also increased in the gonadal AT of $\mathrm{Klf3}^{-/-}$mice (Supplementary Fig. 1d), as were UCP1 and mitochondrial oxphos proteins (Supplementary Fig. 1e, f). Several genes were modestly increased in interscapular brown AT of $\mathrm{Klf3}^{-/}$mice while beige-specific markers ${ }^{1} \mathrm{~Tb} \times 1$, Tmem 26 and Cd137 were undetectable (Supplementary Fig. 1g). UCP1 protein content was mildly decreased in $\mathrm{Klf}^{-/-}$brown AT (Supplementary Fig. 1h, i). While this suggests that brown AT is unlikely to play a major role in the thermogenic phenotype of $\mathrm{Klf3}^{-/}$mice, we cannot wholly rule out its contribution given the existence of UCP1-independent thermogenic mechanisms in beige and brown fat ${ }^{22-25}$. Together, these results show that $\mathrm{Klf3}^{-/}$mice exhibit reduced fat mass that may result from enhanced AT beiging, as demonstrated by the widespread derepression of thermogenic genes and mitochondrial proteins.

KLF3 deficiency enhances the thermogenic response. Cold exposure is a recognised means of promoting adaptive thermogenesis in $\mathrm{AT}^{26-28}$. To study whether the absence of KLF3 enhances beige AT activation and influences body temperature both in the cold and at thermoneutrality, we acutely exposed WT and $\mathrm{Klf3} 3^{-/-}$mice to ambient temperatures of $4{ }^{\circ} \mathrm{C}$ or $30^{\circ} \mathrm{C}$ (Fig. 2a). No statistically significant difference was seen in the body temperatures of WT and $\mathrm{Klf3}^{-/-}$mice during cold exposure (Fig. 2b). As expected, there was little disparity in body temperature between WT and $\mathrm{Klf3} 3^{-1-}$ mice housed at $30^{\circ} \mathrm{C}$. Subcut (Fig. 2c and Supplementary Fig. 3a) and gonadal AT (Supplementary Fig. 3b, c) from Klf3 $3^{-/}$mice were smaller than WT depots under both conditions, and interestingly, interscapular brown AT was smaller in $\mathrm{Klf3} 3^{-/-}$mice acutely exposed to $4{ }^{\circ} \mathrm{C}$ than in WT mice at $4^{\circ} \mathrm{C}$ or in $K l f 3^{-/-}$mice at thermoneutrality (Supplementary Figs. 3d, e). Visual inspection of subcut AT depots from mice housed at $30^{\circ} \mathrm{C}$ and $4{ }^{\circ} \mathrm{C}$ showed that $K l f 3^{-/-}$ mice exhibited distinct, enhanced evidence of browning at $4{ }^{\circ} \mathrm{C}$ compared to WT mice, and this disparity was retained at $30^{\circ} \mathrm{C}$ though to a lesser extent (Fig. 2d). This striking change in macroscopic appearance was surprising, given the short period of cold exposure $(5 \mathrm{~h})$, and may in part be strain-dependent and due to the elevated levels of basal thermogenesis in $\mathrm{Klf3}^{-/}$mice. To examine beige AT activation at the transcriptional level, we assessed expression of thermogenic genes in subcut AT from mice housed at $30^{\circ} \mathrm{C}$ and $4{ }^{\circ} \mathrm{C}$. At $4{ }^{\circ} \mathrm{C}$, we found that multiple genes were increased in the absence of KLF3, including Ucp1, Pgcla, Cidea, Elovl3 and Cpt1b (Fig. 2e), with similar deregulation seen in gonadal AT (Supplementary Fig. 3f) and to a lesser extent, brown AT (Supplementary Fig. 3g). Ucp1 mRNA expression was elevated in subcut AT from $\mathrm{Klf3}^{-1}$ - mice compared to WT mice housed at $30^{\circ} \mathrm{C}$. Western blotting of subcut UCP1 and 
a

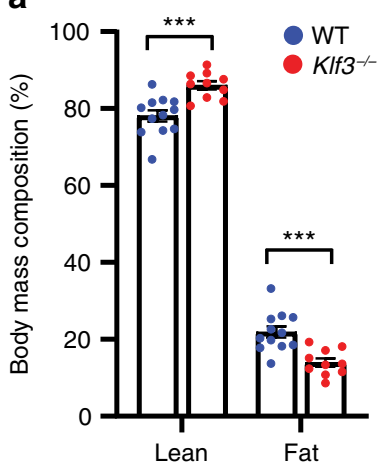

d

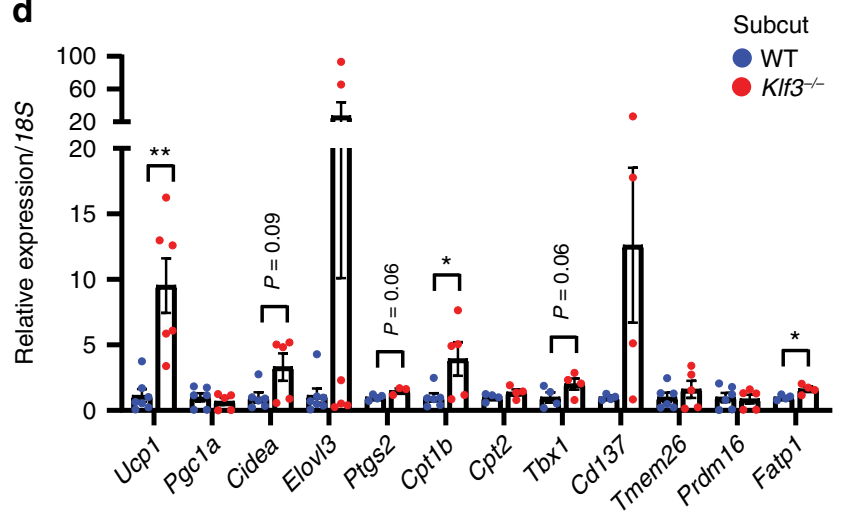

b

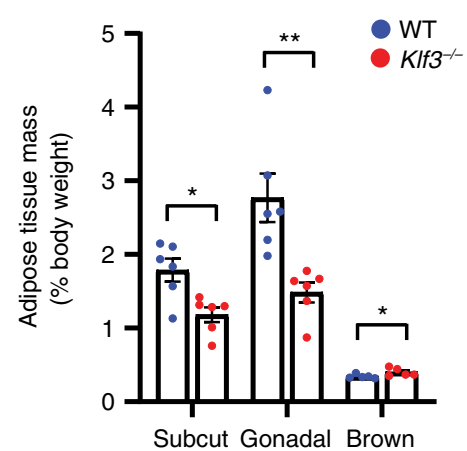

Subcut

WT
C

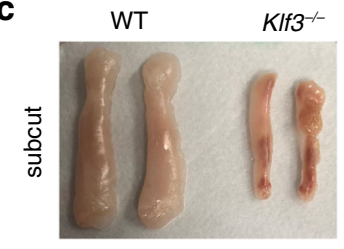

e
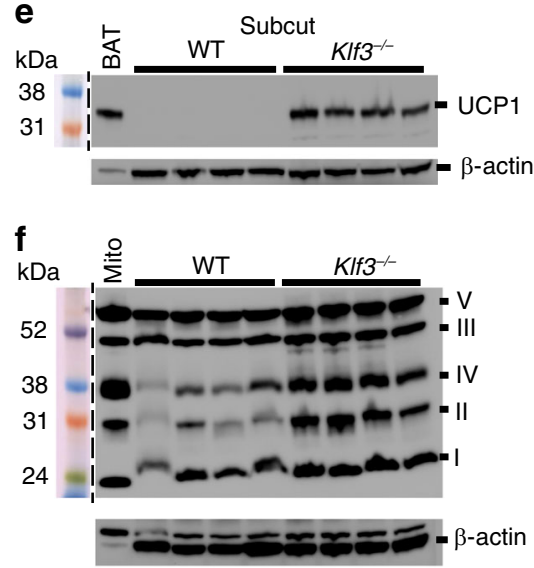

g

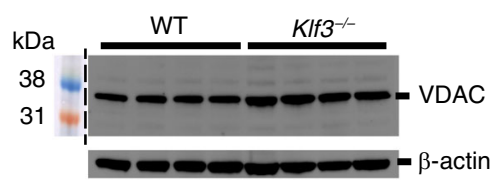

Fig. 1 Reduced adiposity and enhanced beiging in KIf3-/- mice. a Lean and fat body mass composition (\%) of WT $(n=12)$ and $K I f 3^{-/-}(n=10)$ mice were assessed by EchoMRI analysis. $\mathbf{b}$ Weights of WT and KIf3-/- AT depots were recorded as a percentage of total body weight ( $n=6$ mice for subcut and gonadal AT; $n=5$ mice for brown AT). c Representative macroscopic images of WT and KIf3-1- subcut AT pads showing relative size and complexion. $\mathbf{d}$ mRNA levels of thermogenic genes were assessed by qPCR in WT and KIf3-/- subcut AT $(n=4-8$ mice). Relative expression was normalised to $18 \mathrm{~S}$ rRNA levels and the mean WT value for each gene was set to 1. e UCP1 protein expression was measured in WT and KIf3-/- subcut AT by western blotting ( $n=4$ mice). $25 \mu$ of whole-cell extract (WCE) was loaded alongside a Rainbow molecular weight marker. Nitrocellulose membranes were probed with anti-UCP1 antibody overnight. Diluted brown adipose tissue (BAT) extract included as a positive control. $\mathbf{f}$ Expression of mitochondrial oxphos proteins in WT and KIf3-/- subcut AT WCE was assessed by western blotting $(n=4$ mice). $25 \mu \mathrm{g}$ of extract was loaded alongside rat heart mitochondria extract (Mito; positive control), and PVDF membranes were blocked overnight before being probed with the Total OXPHOS Rodent WB Antibody Cocktail for $2 \mathrm{~h}$. Mitochondrial complexes are labelled by their Roman numerals (I-V). $\mathbf{g}$ VDAC protein expression was measured in WT and KIf3-/- subcut AT ( $n=4$ mice) by western blotting. $20 \mu \mathrm{g}$ of WCE was loaded and nitrocellulose membranes were probed overnight with anti-VDAC antibody. For $\mathbf{e}-\mathbf{g} \beta$-actin was included as a loading control. For $\mathbf{a}, \mathbf{b}$ and $\mathbf{d}$, error bars represent means $\pm \mathrm{SEM}$ and one-sided non-parametric Mann-Whitney $U$ tests were performed where ${ }^{\star} P<0.05,{ }^{\star \star} P<0.01,{ }^{\star \star \star} P<0.001$. Source data are provided as a Source data file. $k D a, k i l o d a l t o n s$. See also Supplementary Figs. 1 and 2.

mitochondrial oxphos complexes confirmed upregulation at the protein level at $4^{\circ} \mathrm{C}$ (Fig. $2 \mathrm{f}$, g). Thus, under thermal stress at $4^{\circ} \mathrm{C}$, activation of beige AT is amplified in $\mathrm{Klf3} 3^{-/-}$mice. At thermoneutrality, KLF3 deficiency still augments beige AT activation, suggesting that the effects seen are not solely due to cold temperature, but that in this model beiging of white AT can occur independently of thermal stress.

Reduced weight gain in WT mice transplanted with $\mathrm{Klf3}^{-1-}$ BM. We have previously shown that mice lacking KLF3 are protected from obesity when fed a high-fat diet, with no difference exhibited in food intake on either a chow or high-fat diet compared to WT mice ${ }^{12}$. While the mechanisms underlying had remained unclear, evidence of enhanced beiging in $\mathrm{Klf}^{-/-}$mice now provides a possible explanation. The role of haematopoietic cells in AT function and thermogenesis is now widely appreciated, and KLF3 is both active in haematopoietic lineages ${ }^{29-33}$ and highly expressed in a range of haematopoietic cell types according to the Immgen ${ }^{34}$ and Haemopedia ${ }^{35,36}$ databases. We thus focused on a possible role for KLF3 in BM haematopoietic cells that could give rise to cells occupying the AT SVF niche and regulate adipose function. We irradiated WT mice and transplanted them with BM from WT or $\mathrm{Klf3}^{-1-}$ mice to produce $\mathrm{WT}^{\mathrm{WT}}$ and $\mathrm{WT}^{\mathrm{Klf} 3-/}-$ chimeras (Fig. 3a). Chimeric mice and a control cohort of untransplanted WT and $\mathrm{Klf3}^{-/-}$animals were fed a high-fat, high-sugar Western diet for 11 weeks in order to investigate resistance to diet-induced obesity and adiposity. Successful haematopoietic reconstitution was assessed by genotyping tail biopsies (recipient and donor cells) and BM (recipient cells only) (Supplementary Fig. 4a). Prior to commencing the Western diet regimen, WT ${ }^{K l f 3-/-}$ chimeras were heavier than $\mathrm{WT}^{\mathrm{WT}}$ counterparts, then over the 11-week Western diet period, WTKlf3-l- chimeras gained modestly but significantly less weight than WTWT counterparts (Fig. 3b and Supplementary Fig. 4b, c) and had less body fat (Fig. 3c). Importantly, WT Klf3-/- chimeras gained less subcut AT mass compared to $\mathrm{WT}^{\mathrm{WT}}$ animals (Fig. $3 \mathrm{~d}$ and Supplementary Fig. 4d) and more prominent browning in WTKlf3-l- subcut AT was detected by visual inspection (Fig. 3e). No such differences were observed in the weights or appearance of gonadal or brown AT (Supplementary Fig. 4e-i). 
a

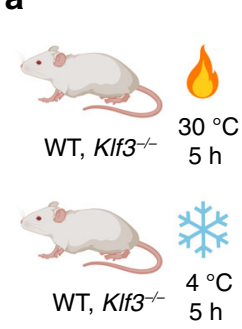

b

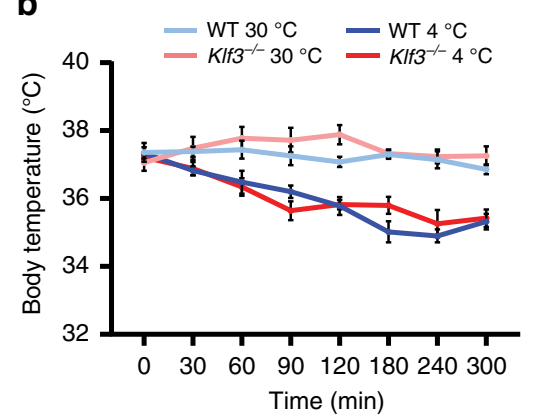

C

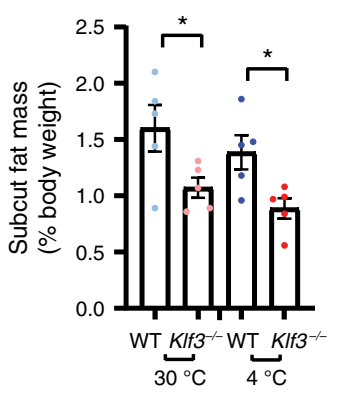

d

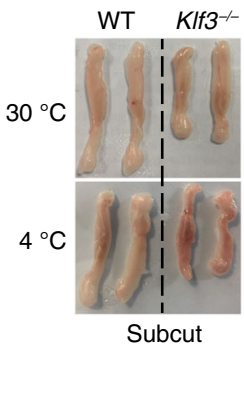

f

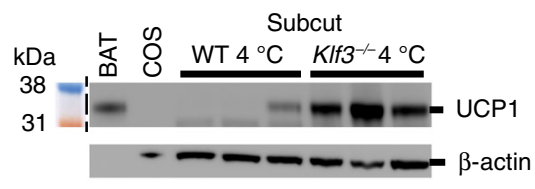

g

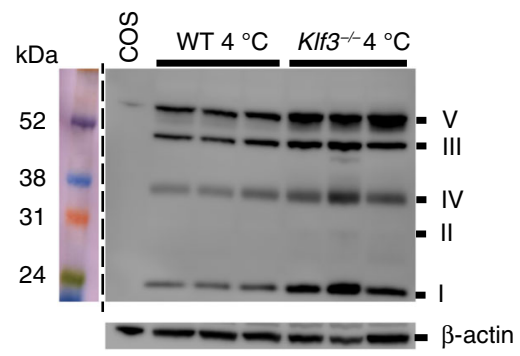

Fig. 2 KLF3 deficiency enhances the thermogenic response. a WT and $K I f 3^{-/-}$mice were acutely housed at $30^{\circ} \mathrm{C}$ or $4^{\circ} \mathrm{C}$ (cartoon created with BioRender.com) and $\mathbf{b}$ body temperature was monitored by rectal probing over a $5 \mathrm{~h}$ period $\left(n=5\right.$ mice). c Weights of WT and KIf $3^{-/-}$subcut AT from mice housed at $30^{\circ} \mathrm{C}$ or $4^{\circ} \mathrm{C}$ were recorded as relative to body weight $(n=5$ mice.) d Representative macroscopic images of subcut AT from WT and $\mathrm{KIf3}^{-/-}$mice housed at $30^{\circ} \mathrm{C}$ or $4^{\circ} \mathrm{C}$, showing size and complexion. e mRNA levels of thermogenic genes were assessed by qPCR in WT and KIf3- ${ }^{-}$ subcut AT ( $n=5$ mice). Relative expression was normalised to $18 \mathrm{~S}$ rRNA levels and the WT $30^{\circ} \mathrm{C}$ value for each gene was set to $1 . \mathbf{f}$ UCP1 protein expression was measured in subcut AT from WT and $K I f 3^{-/-}$mice housed at $4^{\circ} \mathrm{C}$ by western blotting $(n=3$ mice $) .25 \mu \mathrm{g}$ of WCE was loaded alongside a Rainbow molecular weight marker. Nitrocellulose membranes were probed with anti-UCP1 antibody overnight. $\beta$-actin was included as a loading control and diluted brown AT (BAT) extract included as a positive control. $\mathbf{g}$ Expression of mitochondrial oxphos proteins in subcut AT from WT and KIf3-/- mice housed at $4{ }^{\circ} \mathrm{C}$ was assessed by western blotting $(n=3$ mice). $25 \mu$ g of extract was loaded alongside rat heart mitochondria extract (Mito; positive control), and PVDF membranes were blocked overnight before being probed with the Total OXPHOS Rodent WB Antibody Cocktail for $2 \mathrm{~h}$. $\beta$-actin was including as a loading control. Mitochondrial complexes are labelled by their Roman numerals (I-V). Extracts from COS cells were included as negative control lanes. For $\mathbf{b}$ two-way ANOVA followed by post-hoc Tukey testing was performed to assess statistical significance. For $\mathbf{c}$, e, error bars represent means \pm SEM and one-sided non-parametric Mann-Whitney $U$ tests were performed where ${ }^{\star} P<0.05$, ${ }^{\star} P<0.01$. Source data are provided as a Source data file. kDa, kilodaltons; COS, COS-7 cells. See also Supplementary Fig. 3.

The expression of thermogenic genes in the subcut AT of chimeric mice was tested and Ucp 1, Cpt1b and Cd137 were found to be upregulated in animals that received $\mathrm{Klf}^{-/-}$BM compared to mice that received WT BM (Fig. 3f). Several genes were also upregulated in WTKlf3-/- gonadal and brown AT (Supplementary Fig. 4j, k). We performed biochemical analysis on the peripheral blood of transplanted mice and showed that plasma triglycerides were significantly lower in $\mathrm{WT}^{\mathrm{Klf3} 3-/-}$ mice compared to $\mathrm{WT}^{\mathrm{WT}}$, while no difference existed in total cholesterol (Supplementary Fig. 5a, b). We also detected reduced hepatic triglycerides in untransplanted KLF3-deficient mice (Supplementary Fig. 5c), and observed no difference in liver cholesterol levels (Supplementary Fig. 5d). Together these results demonstrate that transplantation of irradiated WT mice with KLF3deficient BM was sufficient to confer metabolic benefits on recipient mice. This suggests that haematopoietic cells may be instrumental in driving beiging and conferring resistance to obesity in this system.

AT-resident eosinophils are altered in the absence of KLF3. Given that transplantation with KLF3-deficient haematopoietic cells promoted resistance to obesity, we sought to identify the important cell types in this process. KLF3 is expressed in multiple tissues and cell lineages, including various haematopoietic cells $^{34,35}$, and the FANTOM5 SSTAR database that measures transcript levels indicates it is more abundant in human eosinophils than any other cell type ${ }^{37}$, consistent with its observed presence in murine eosinophils ${ }^{38}$ (Supplementary Fig. 6a). Given their proposed role in adiposity and thermogenesis ${ }^{3-5,39-41}$, we sought to assess the contribution of eosinophils in driving the thermogenic phenotype of $K l f 3^{-/-}$mice. We first examined the abundance of eosinophils in subcut AT of WT and $\mathrm{Klf3}^{-/-}$ mice. SVF cells were collected and analysed by flow cytometry (according to the eosinophil gating strategy in Supplementary Fig. 7a). AT-resident eosinophils were 3 -fold more prevalent in $\mathrm{Klf}^{-1-}$ subcut AT (Fig. 4a). We also sorted eosinophils from whole subcut AT to determine the number of cells per gram and confirmed that eosinophil abundance is greater in $\mathrm{Klf}^{-/-}$ subcut AT (Fig. 4b). mRNA levels of the gene encoding the eosinophil surface marker Siglec-F (Siglecf) were also 3-fold higher in $\mathrm{Klf}^{-/-}$subcut AT (Fig. 4c). It is noteworthy that while eosinophils are more than 2-fold more abundant in $\mathrm{Klf3}^{-/-}$ 
a

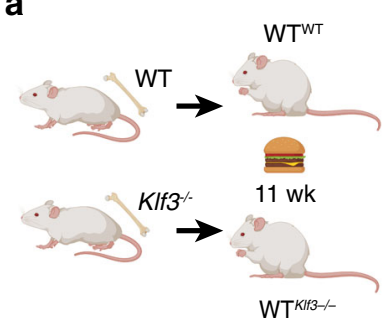

b

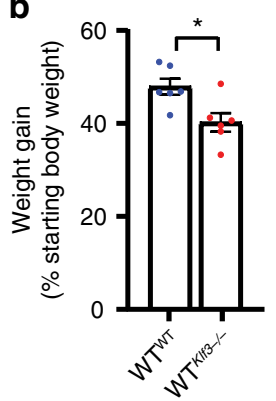

d

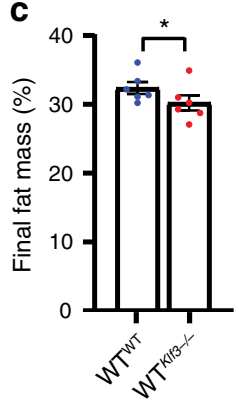

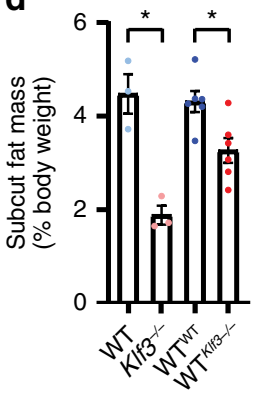

e

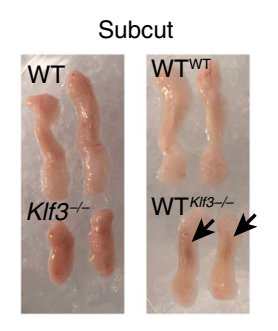

$\mathbf{f}$

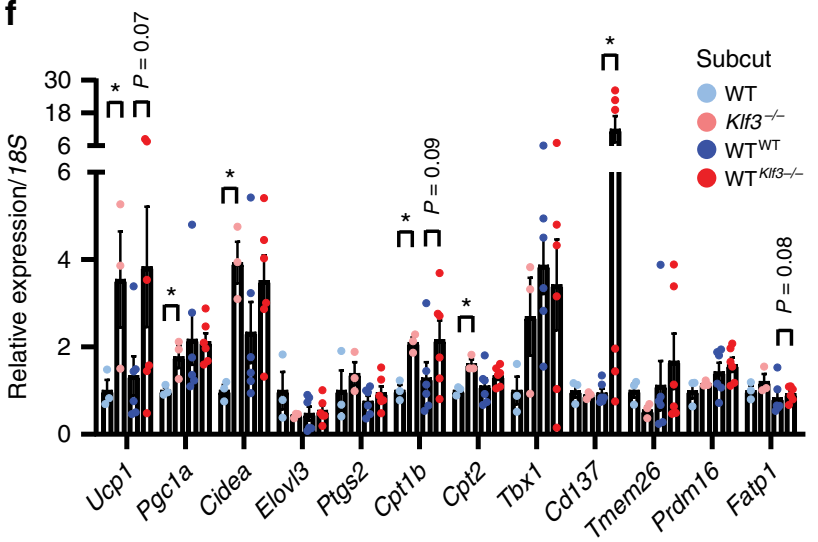

Fig. 3 Reduced weight gain in WT mice transplanted with $\mathbf{K I f 3}^{-/-}$BM. a WT mice were irradiated and transplanted with bone marrow from WT (WTWT) or KIf3-/- (WTKIf3-/-) donors then fed a high-fat, high-sugar (Western) diet for 11 weeks (wk) (cartoon created with BioRender.com). b Body weight was monitored throughout the Western diet study duration (weeks 2-13), and the total weight gained was calculated as a percentage of starting body weight at commencement of Western diet administration ( $n=6$ mice). c Fat mass composition (\%) was assessed using EchoMRI after 11 weeks of Western diet ( $n=6$ mice). d Weights of subcut AT (relative to body weight) from WT, KIf3 ${ }^{-/-}(n=3)$, WTWT and WTKIf3- $/-(n=6)$ mice fed a Western diet for 11 weeks. e Representative macroscopic images of subcut AT pads from mice of each condition, showing size and complexion. Arrows point to sites of enriched brown appearance. $\mathbf{f}$ mRNA levels of thermogenic genes in the subcut AT of WT, KIf3-/- $(n=3)$, WTWT and WTKIf3-/- $(n=6)$ mice were assessed by qPCR. Relative expression was normalised to levels of $18 \mathrm{~S}$ rRNA and the mean WT value set to 1 for each target. For $\mathbf{b}$-d and $\mathbf{f}$, error bars represent means \pm SEM and one-sided non-parametric Mann-Whitney $U$ tests were performed where ${ }^{\star} P<0.05$. Source data are provided as a Source data file. See also Supplementary Figs. 4, 5.

gonadal AT (Supplementary Fig. 7b), no differences in the number of lung or spleen eosinophils were detected (Supplementary Fig. 7c, d). Eosinophils were also found to be over 2 -fold more prevalent in the subcut AT of $\mathrm{WT}^{\mathrm{Klf3} 3-/-}$ chimeric mice than in $\mathrm{WT}^{\mathrm{WT}}$ counterparts on a Western diet (Supplementary Fig. 7e, f). We next compared the genome-wide expression profiles of eosinophils isolated from subcut SVF by FACS and observed broad deregulation of gene expression in the absence of KLF3 (Fig. 4d), resulting in changes to important cellular pathways including signalling, localisation, response to stimulus and immune system processes (Supplementary Fig. 6b). Further breakdown of the deregulated immune system processes in $\mathrm{Klf3}^{-/-}$eosinophils revealed striking enrichment of various biological pathways such as leukocyte migration and myeloid cell homeostasis (Supplementary Fig. 6c). To explore the transcriptional network further we interrogated the transcriptional activator $K l f 1$, which operates in a regulatory system with $K l f 3$ in erythroid cells ${ }^{32,42}$. Klf1 was very lowly expressed in AT eosinophils and showed no difference between WT and $\mathrm{Klf3}^{-/-}$(Supplementary Fig. 6d, e), with no expression detected in whole AT or SVF (Supplementary Fig. 6f), suggesting that KLF1 does not play an important opposing role to KLF3 in AT eosinophils. Inspection of the most significantly deregulated genes in KLF3deficient adipose eosinophils revealed three genes encoding proteins with reported roles in beige AT activation-meteorin-like $(\text { Metrnl })^{5}$, met-enkephalin $(\text { Penk })^{14,16,43}$ and IL-33 (Il33) $)^{14,15}$
(Fig. 4e). We have previously reported that levels of galectin-3, a proposed eosinophil chemoattractant ${ }^{44,45}$, are elevated in $\mathrm{Klf3}^{-/-}$ $\mathrm{AT}^{29}$. Here we found that the eosinophil chemoattractants eotaxin-1 and -2 (Ccl11 and Ccl24) were upregulated in KLF3deficient AT eosinophils (Fig. 4f), providing a possible explanation for the increased eosinophil abundance in $\mathrm{Klf3}^{-/}$AT. Given that eosinophil abundance is elevated in $\mathrm{Klf}^{-/-} \mathrm{AT}$, we sought to quantify other immune cells in the stromal vascular compartment. We did not observe changes to macrophage abundance (M1 or M2), dendritic cells, CD19 ${ }^{+} \mathrm{B}$ cells or $\mathrm{CD}^{+} \mathrm{T}$ cells (Fig. 4g, h and Supplementary Fig. 8a-e). T regulatory lymphocytes (Tregs) were more abundant in KLF3-deficient subcut AT (Fig. 4i and Supplementary Fig. 8a)-unsurprising given the elevated expression of IL-33 ${ }^{46-49}$. While no significant differences to the overall lineage ${ }^{-} \mathrm{CD} 127^{+} \mathrm{CD} 25^{+}$group 2 innate lymphoid cell (ILC2) population were observed, we did detect a significant deficit of ILC2s expressing the IL-33 receptor (ST2) in $\mathrm{Klf3}^{-/-}$ subcut AT (Fig. 4j and Supplementary Fig. 8a, f, g). To assess whether changes in the AT immune profile affected cytokine signalling, we measured expression of various inflammatory and anti-inflammatory genes in the SVF of WT and $\mathrm{Klf3}^{-/-}$subcut AT. Expression of the classical inflammatory marker $\operatorname{Tnf}$ (encoding the pro-inflammatory molecule TNF- $\alpha$ ) was reduced in the absence of KLF3, while the M2 macrophage marker Arg1 was increased (Supplementary Fig. 7g). Unexpectedly, we detected increased expression of Ifng (encoding the inflammatory cytokine 
a

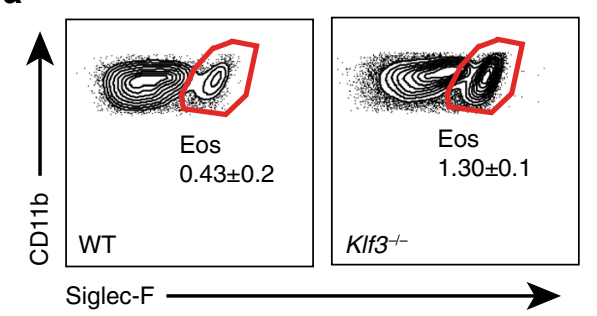

b

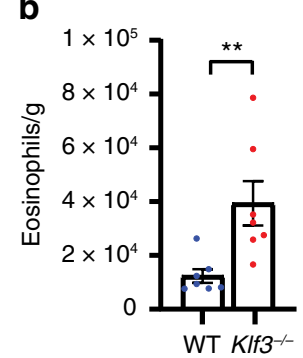

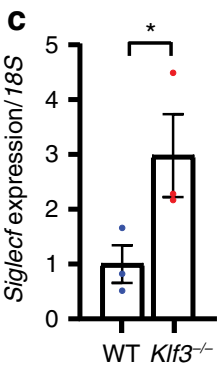

d
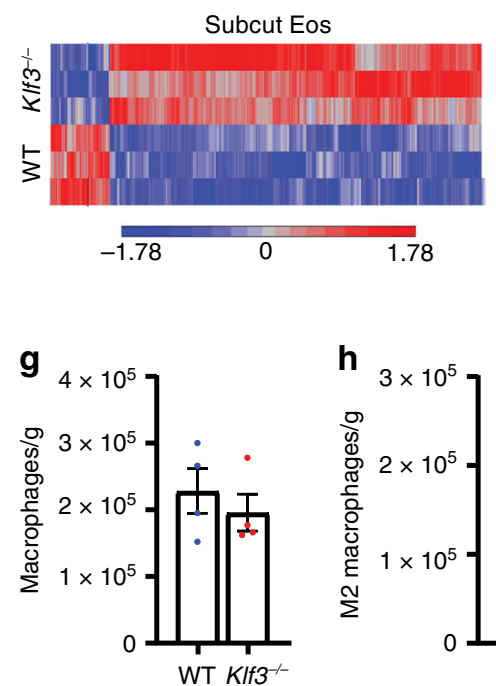

e
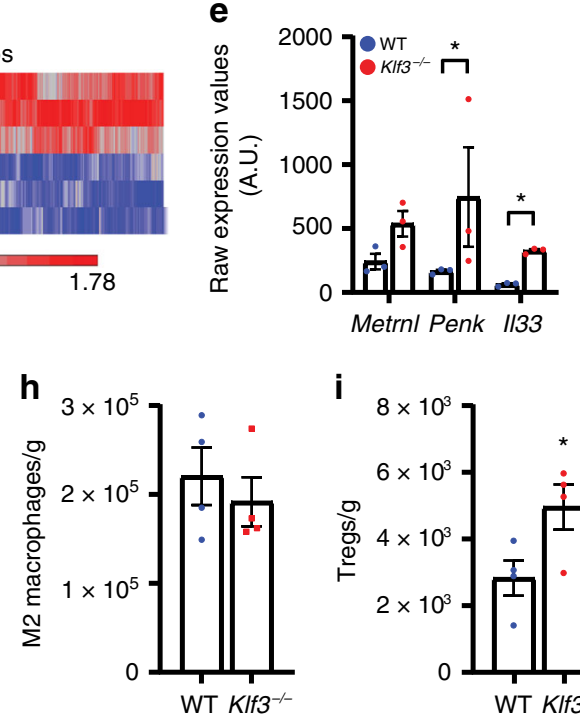

i

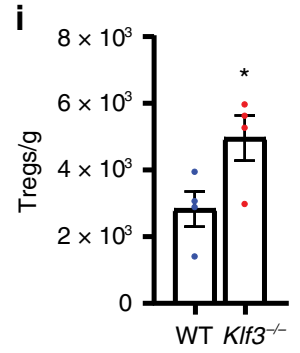

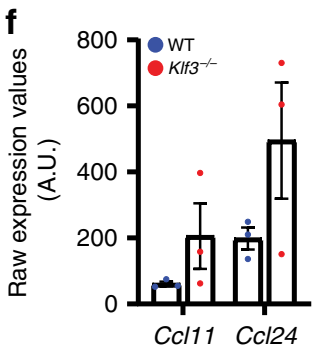

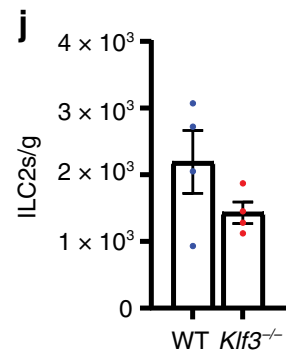

Fig. 4 AT-resident eosinophils are altered in the absence of KLF3. a Flow cytometry was used to analyse the abundance of Siglec- $\mathrm{F}^{+}$eosinophils in the SVF of WT and $\mathrm{KIf3}^{-/-}$subcut AT. Representative plots of Siglec- $\mathrm{F}^{+}$eosinophils as a percentage of live cells are shown accompanied by the means \pm SEM of $n=3$ mice per genotype. $\mathbf{b}$ The total number of eosinophils per gram of subcut AT was calculated by sorting the entire SVF from WT and KIf3-/- mice ( $n=7$ mice). c mRNA levels of the murine eosinophil surface marker Siglecf were assessed by qPCR ( $n=3$ mice). Relative expression was normalised to levels of $18 \mathrm{~S}$ rRNA. d Eosinophils isolated from the subcut SVF of WT and KIf3-/- mice by FACS were subjected to RNA extraction for microarray analysis, available at GEO (Accession No. GSE117445) ( $n=3$ mice). A heatmap was constructed showing significantly deregulated genes $(P<0.05$, two-way ANOVA using false discovery rate, FDR, to correct for multiple comparisons). e Three genes with reported roles in beige fat activation and AT homeostasis were identified as significantly upregulated in $K I f 3^{-/-}$AT eosinophils and their raw expression values were calculated by robust multi-array average (RMA) normalisation, with error bars representative of the means \pm SEM of $n=3$ mice per genotype. $\mathbf{f}$ Raw expression values for eotaxins $C c / 11$ and $C c / 24$

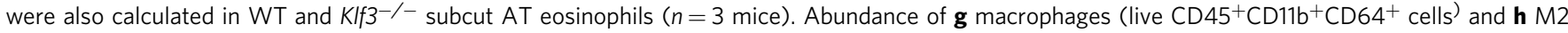
macrophages (live $\mathrm{CD} 45^{+} \mathrm{CD} 11 \mathrm{~b}^{+} \mathrm{CD} 64^{+} \mathrm{CD} 206^{+} \mathrm{CD}_{11 c^{-}}$cells) in the SVF of WT and Klf3-/- subcut AT was determined by flow cytometry and normalised to tissue weight $\left(n=4\right.$ mice). Abundance of $\mathbf{i}$ Tregs (live CD45+CD19-CD3+CD4+CD25+ cells) and $\mathbf{j} / \mathrm{LC} 2 \mathrm{~s}\left(\mathrm{CD} 45^{+} \mathrm{Lin}^{-} \mathrm{CD} 127^{+} \mathrm{CD} 25^{+}\right.$ cells) were also determined by flow cytometry and normalized to tissue weight $(n=4$ mice). For $\mathbf{b}, \mathbf{c}$ and $\mathbf{e}-\mathbf{j}$ error bars represent means \pm SEM and onesided non-parametric Mann-Whitney $U$ tests were performed where ${ }^{\star} P<0.05,{ }^{\star} P<0.01$. Source data are provided as a Source data file. Eos, eosinophils; Tregs, regulatory T cells; ILC2s, group 2 innate lymphoid cells; A.U., arbitrary units. See also Supplementary Figs. 6-8.

interferon- $\gamma$ ) in $\mathrm{Klf3}^{-/-}$, which has been shown to block themogenesis ${ }^{50}$. On the other hand, levels of $I l 10$ were reduced in the absence of KLF3, in line with recent findings that IL-10 blockade protects against diet-induced obesity and elicits browning of $\mathrm{AT}^{51}$. Together these findings demonstrate that resident eosinophils are more abundant in $\mathrm{Klf3}^{-/-} \mathrm{AT}$ and that these cells exhibit widespread deregulated gene expression, including higher levels of genes implicated in beige AT activation. These changes are accompanied by modifications to the immune and cytokine landscape of subcut AT.

KLF3 directly regulates AT eosinophil gene expression. Our finding that AT eosinophils lacking KLF3 express higher levels of Metrnl, Penk and Il33 led us to examine these genes more closely. Using qPCR analysis, we measured higher expression of Metrnl and Il33 in subcut SVF from $\mathrm{Klf3}^{-/-}$compared to WT mice (Fig. 5a). Likewise, we found that levels Metrnl and Il33 were

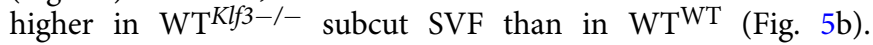

Interestingly, Penk expression was only elevated in subcut SVF from $\mathrm{Klf}^{-}{ }^{-}$- mice fed a Western diet, with no differences seen in whole subcut AT or SVF from transplanted mice or from mice fed a chow diet (Fig. 5a, b and Supplementary Fig. 8a, b). To assess levels of secreted meteorin-like and IL-33 in AT, we quantified their concentration in supernatant from AT explants cultured for $2 \mathrm{~h}$, using ELISA. We found that both meteorin-like and IL-33 were more highly concentrated in Klf3 ${ }^{-/}$AT supernatant, suggesting greater secretion of these proteins (Fig. $5 c, d$ ). However, when we measured meteorin-like and IL-33 in peripheral blood plasma we found no difference (Supplementary Fig. 8c, d), which may suggest that these proteins are elevated locally and have a paracrine effect confined to the AT microenvironment. To determine whether AT eosinophils are able to directly drive beiging of adipocytes, we performed co-culture experiments. FACS-sorted eosinophils from WT and $\mathrm{Klf3}^{-/-}$ subcut SVF were co-cultured with mature primary adipocytes for $4 \mathrm{~h}$, and the expression of thermogenic genes measured by 

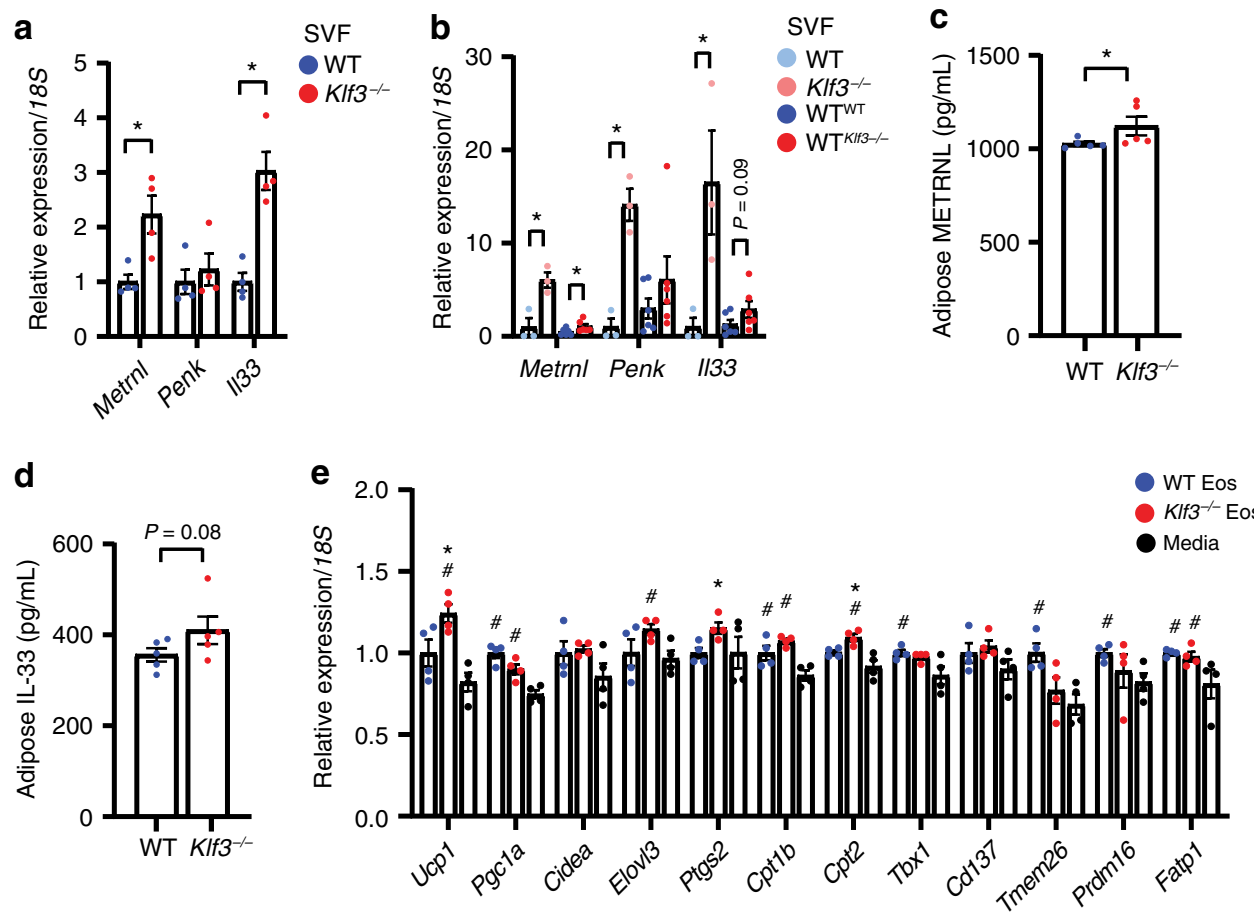

f

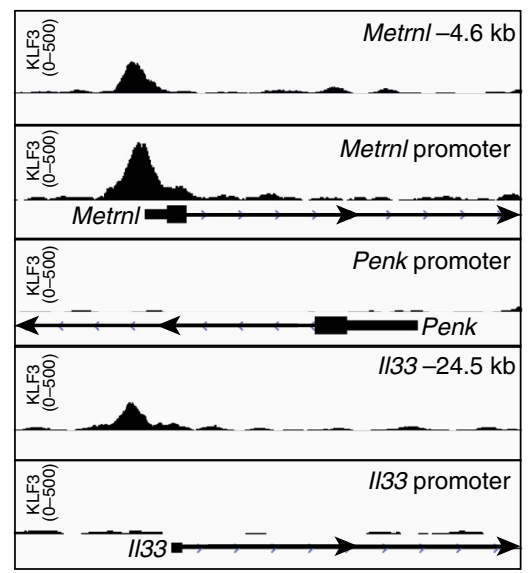

g

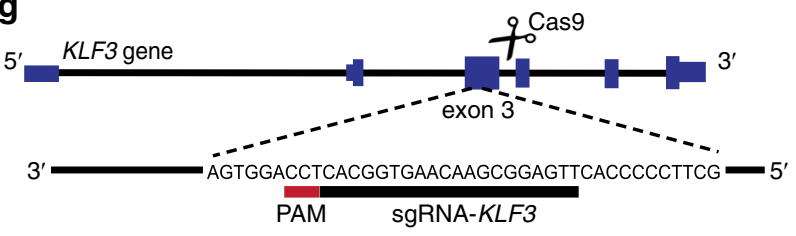

h

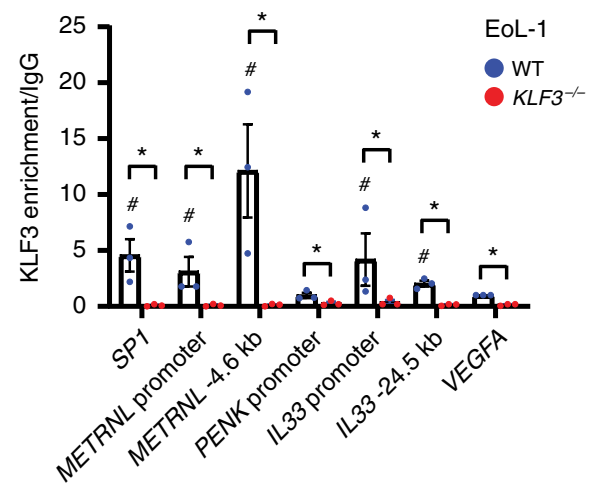

Fig. 5 KLF3 directly regulates AT eosinophil gene expression. a Metrnl, Penk and $I / 33$ mRNA levels in subcut SVF from a WT and $K I f 3-/-$ mice $(n=4$ mice) and $\mathbf{b} \mathrm{WT}, \mathrm{KIf3}^{-/-}(n=3), \mathrm{WTWT}$ and WTKIf3-/- $(n=6)$ mice. Relative expression was normalised to $18 \mathrm{~S}$ rRNA and WT values set to 1 for each gene. Levels of secreted $\mathbf{c}$ meteorin-like and $\mathbf{d}$ IL-33 were measured in supernatants of WT and KIf3-/- AT explants by ELISA ( $n=5$ mice). e Expression of thermogenic genes in differentiated preadipocytes co-cultured for $4 \mathrm{~h}$ with WT or KIf3- $/$ - eosinophils (or media alone) was measured by qPCR ( $n=4$ biological replicates). Relative expression was normalised to $18 \mathrm{~S}$ and WT eosinophil-treated values set to 1. $\mathbf{f}$ Tracks at the promoter and upstream regions of Metrnl, Penk and II33 were obtained from a KLF3-V5 ChIP-Seq in murine embryonic fibroblasts (GEO Accession No. GSE44748) to assess in vivo KLF3 binding. Scale is shown on the left (KLF3 0-500) and regions are denoted in the top right corner. $\mathbf{g}$ CRISPR/Cas9 strategy used to delete KLF3 in EoL-1 cells. h ChIP-qPCR was performed in WT and KLF3-/- human EoL-1 cells to assess direct binding of KLF3 to Metrnl, Penk and II33 ( $n=3$ independent experiments). KLF3 enrichment is normalised to input (non-immunoprecipitated DNA) and a goat IgG control, with human SP1 and VEGFA included as positive and negative control loci respectively. KLF3-/- EoL-1 cells were used to confirm the affinity of the anti-KLF3 antibody to KLF3 antigen, with no enrichment expected in these cells. For a-d one-sided non-parametric Mann-Whitney $U$ tests were performed where ${ }^{\star} P<0.05$. For e one-sided non-parametric Mann-Whitney $U$ tests were performed where ${ }^{*} P<0.05$ between WT and KIf $3^{-/-}$eosinophil co-culture, and ${ }^{\#} P<0.05$ between WT or KIf3-/- eosinophil co-culture and media alone treatment. For $\mathbf{h}$ one-sided non-parametric Mann-Whitney $U$ tests were performed where ${ }^{\star} P<0.05$ between WT and $K L F 3^{-1-}$ for each region and \#P<0.05 relative to the WT VEGFA negative control. For $\mathbf{a}-\mathbf{e}$ and $\mathbf{g}, \mathbf{h}$, error bars represent means \pm SEM. Source data are provided as a Source data file. Eos, eosinophils; kb, kilobase pairs; PAM, protospacer adjacent motif. See also Supplementary Fig. 9. 


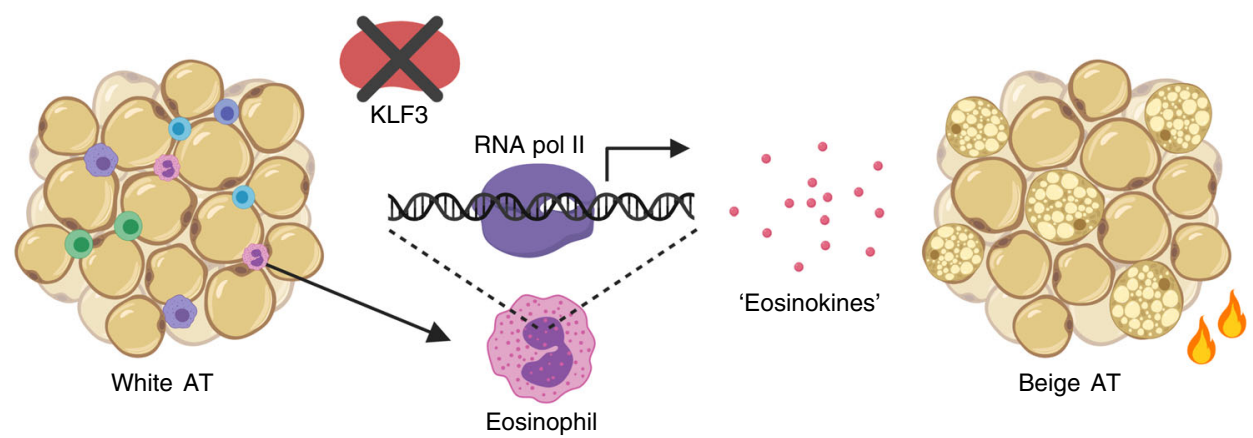

Fig. 6 KLF3 regulation of eosinophil gene expression and function in adipose tissue. Proposed model by which KLF3 may regulate the expression of eosinophil-derived factors in the conversion from white to beige AT.

qPCR (Fig. 5e). We observed modest but significant increases in the expression of several thermogenic genes in adipocytes cocultured with WT eosinophils compared to media alone. Additionally, several genes, including Ucp1, Ptgs 2 and Cpt2, were more highly expressed in adipocytes co-cultured with $\mathrm{Klf3}^{-/-}$AT eosinophils than WT eosinophils. Together these results suggest that AT eosinophils alone are able to drive beiging of adipocytes, at least in vitro, and that KLF3 may directly regulate gene expression in eosinophils. Given that in the absence of the transcriptional repressor KLF3, Metrnl and Il33 are upregulated and secreted in higher abundance within AT, we sought to determine whether KLF3 directly binds and regulates these genes. Inspection of ChIP-Seq data from mouse embryonic fibroblasts (Accession No. GSE44748) ${ }^{52}$ showed distinct KLF3 binding at the Metrnl promoter region, but little enrichment at Penk or Il33 (Fig. 5f). To assess whether KLF3 binds these genes in a relevant cellular setting we performed ChIP in WT and $K L F 3^{-/-}$EoL-1 cells, a human eosinophilic cell line that highly expresses KLF3 (Supplementary Fig. 8e). KLF3-/- cells were generated by CRISPR/Cas9 non-homologous end joining following the introduction of a sgRNA targeting exon 3 of KLF3 (Fig. 5g). After undertaking immunoprecipitations with an anti-KLF3 antibody or normal goat IgG, we performed qPCR using primers designed to amplify the promoter regions of METRNL, PENK and IL33, and to positive control regions based on known ChIP-Seq peaks (SP1, METRNL $-4.6 \mathrm{~kb}$, IL33 $-24.5 \mathrm{~kb})$ and a negative control locus (VEGFA). We found KLF3 binding was evident at the $M E T R N L$ and IL33 promoters compared to the negative control region $V E G F A$, and that signals in $K_{L F 3}{ }^{-/-}$samples were negligible, confirming the specificity of the anti-KLF3 immunoprecipitation (Fig. 5h). This indicates that KLF3 directly binds and regulates the expression of the genes encoding important secreted molecules in adipose-resident eosinophils.

\section{Discussion}

Type 2 immune cells are now recognised as key players in AT homeostasis and beiging. Much attention has been given to the contribution of macrophages $4,8,53,54$, however recent findings suggest that AT macrophages may not perform their previouslydefined direct contribution to adaptive thermogenesis via catecholamine production ${ }^{9-11}$. This realisation provides us with an opportunity to re-evaluate the current model of type 2 immunity in adiposity and beiging by defining previously unknown or under-appreciated immune cells and the mechanisms underpinning their contribution to AT homeostasis. A recent study found that AT immune cells secrete acetylcholine that elicits beiging, highlighting the importance of haematopoietic cells in AT homeostasis ${ }^{55}$. Interestingly though, acetylcholine-producing cells were predominantly B cells, T cells and macrophages, leaving the contribution of other lineages, such as eosinophils, unresolved. In this study, using a combination of in vitro approaches, transgenic mice and BM transplantation models, we have shown that the transcriptional repressor KLF3 directly regulates genes encoding secreted factors in AT eosinophils. Our findings from co-culture experiments suggest that eosinophils may be able to directly drive activation of beiging in adipocytes through paracrine signalling. This highlights the importance of eosinophils in our model of type 2 immunity in AT beiging and suggests that eosinophils may be able to activate beige fat in an organismal setting, providing protection from obesity. Future work will seek to further characterise the in vivo contribution of eosinophils to adipose homeostasis and thermogenesis, and to understand the relevance of these cells in human setting.

BM transplantation studies have been utilised to explore the contribution of haematopoietic cells in adiposity and beiging, including the metabolic response to caloric restriction ${ }^{56}$ and hypermetabolism following burn injury ${ }^{57}$. We cannot exclude the influence of other haematopoietic cells to the reduced weight gain and improved metabolic parameters in $\mathrm{WT}^{\mathrm{Klf3}-/-}$ mice, and while this work explores the contribution of eosinophils, considerable effort in the field is currently being directed towards understanding the diverse roles of various immune cell populations in beiging. Although eosinophil infiltration has been implicated in beiging 3,40 , it is also apparent that artificially increasing their abundance by administration of IL-5 does not appear to significantly improve major metabolic parameters in diet-induced obese mice ${ }^{7}$. The authors proposed that eosinophil activity may be more important than total numbers in AT. Significantly, as well as observing increased eosinophil abundance in $\mathrm{Klf3}^{-1-} \mathrm{AT}$, we found considerable deregulation of biological pathways in these cells and upregulation of genes encoding key secreted factors that activate beige AT. Meteorin-like is secreted by exercised muscle and induced in AT following cold exposure ${ }^{5}$, however, a comprehensive understanding of the AT-specific source has remained elusive. Recent studies have reported adipose stromal cells as the primary source of IL-33, with only a small contribution potentially originating from hematopoietic cells 48,49 . Here we provide evidence that eosinophils may contribute a portion of local adipose IL-33, as well as meteorin-like. While the absence of Penk deregulation in chow-fed $\mathrm{Klf}^{-/-}$mice and lack of evidence for direct binding by chromatin immunoprecipitation led us to emphasize other putative target genes, we cannot rule out a direct role for KLF3 in the regulation of Penk, or a role for Met-Enk in contributing to the phenotype observed-potentially in other cell types.

Overall, our findings demonstrate a mechanism by which type 2 immune cells may influence beiging and adiposity-via KLF3-driven regulation of important eosinophil-derived factors that we term 'eosinokines' 40 , as shown in our proposed model (Fig. 6). Our results not only suggest that eosinophils may play an 
under-appreciated role as signalling cells in metabolism, but also reveal that KLF3 is a key regulator of secreted molecules in eosinophils. Multiple KLF family members have been implicated in the control of metabolism ${ }^{58-62}$ but few clear unifying models of action have emerged. KLF3, for instance, does not appear to regulate meteorin-like or IL-33 in other cell types, and indeed its role in eosinophils has previously been unrecognised. Targeting molecules secreted by immune cells and their receptors may have the potential to therapeutically drive energy expenditure via AT beiging to combat obesity. Our findings advance the search for secreted factors produced by eosinophils and other resident haematopoietic cells, and support the hypothesis that eosinophils and their secreted 'eosinokines' are relevant to the type 2 immune network in AT.

\section{Methods}

Animal husbandry. All animal work was carried out in accordance with approval from the UNSW Animal Care and Ethics Committee (Approval Nos. 12/150A, 16/ 5B and 16/141B), the Murdoch Children's Research Institute Animal Ethics Committee (Approval No. A760) and the University of Sydney Animal Care and Ethics Committee (Approval No. L02/7-2009/3/5054). Animals were housed in a specific pathogen-free environment at a constant ambient temperature of $22^{\circ} \mathrm{C}$ and $50 \%$ humidity on a $12 \mathrm{~h}$ light-dark cycle, with ad libitum access to standard chow food and water, unless otherwise specified. Generation of global $\mathrm{Klf}^{-/-}$mice on an $\mathrm{FVB} / \mathrm{NJ}$ background has been previously reported ${ }^{13}$. Age-matched WT and $\mathrm{Klf3}^{-/-}$male littermates derived from $\mathrm{Klf3}^{+/-} \times \mathrm{Klf3}^{+/-}$crosses were used for all animal studies. Male mice were housed in cages containing bedding, nesting material and enrichment with up to five individual animals, except for cold and thermoneutral experiments which were undertaken in empty cages containing singly housed mice.

Acute thermoneutral and cold temperature exposure. For acute temperature exposure experiments, WT and $\mathrm{Klf3}^{-1-}$ mice aged between 12 and 14 weeks were assessed at $30^{\circ} \mathrm{C}$ or $4{ }^{\circ} \mathrm{C}$ for $5 \mathrm{~h}$. Mice housed at thermoneutrality $\left(30^{\circ} \mathrm{C}\right)$ were acclimatised at this temperature for $20 \mathrm{~h}$ prior to commencement of experiments. Food, water and bedding were removed from cold-exposed mice during the $5 \mathrm{~h}$ period. Body temperatures were obtained by rectal probe (BAT-12 microprobe thermometer) over a $5 \mathrm{~h}$ period between the times of $0800 \mathrm{~h}$ and $1400 \mathrm{~h}$. Temperatures were measured at 0,30,60, 90, 120, 180, 240 and $300 \mathrm{~min}$. Body weights were recorded before and after the $5 \mathrm{~h}$ temperature measurement period. At the conclusion of the $5 \mathrm{~h}$ assessment, all mice were euthanised and tissues and blood were collected for further analysis.

Bone marrow transplantation studies. WT mice aged 7 weeks old were irradiated using an X-RAD 320 with two doses of $500 \mathrm{cGy}, 6 \mathrm{~h}$ apart. The following day, 7week old WT and $\mathrm{Klf3}^{-1-}$ donor mice were euthanised and femora and tibiae harvested. BM cells were flushed with sterile RPMI medium and red blood cells lysed using distilled and deionised water. Cells were resuspended in phosphatebuffered saline (PBS) and adjusted to $5 \times 10^{7} / \mathrm{mL}$. Recipient mice were warmed using infrared heat lamps then $1 \times 10^{6}$ cells were injected into the tail vein. Transplantation recipients were administered with antibiotics containing $200 \mathrm{mg} / \mathrm{L}$ sulfamethoxazole and $40 \mathrm{mg} / \mathrm{L}$ trimethoprim via drinking water for 2 weeks. Following this, recipient mice and a control cohort of WT and $\mathrm{Klf3}^{-/-}$mice were transferred to a high-fat, high-sugar Western diet for 11 weeks (Supplementary Table 1). Mice were weighed and assessed for body mass composition using an EchoMRI before and throughout the study, and at the conclusion tissues and blood were collected for further analyses. Genotyping of tail biopsies and BM was performed to confirm reconstitution of WT or Klf3 $3^{-1-}$ cells in the BM of chimeric mice. Genotyping primers can be found in Supplementary Table 2, and gel images were analysed using Bio-Rad Image Lab software v6.0.1.

Blood and plasma analysis. Peripheral blood was collected by cardiac bleed using $50 \mathrm{U} / \mathrm{mL}$ heparin sulphate as an anti-coagulant, and stored in K2EDTA collection tubes. Whole blood was sent for biochemical analysis of triglycerides and total cholesterol at the University of Sydney Veterinary Pathology Diagnostic Services. Plasma was isolated by centrifugation $(2000 \times g$ for $15 \mathrm{~min})$ of whole blood and stored for further analysis.

Mouse tissue processing. AT was harvested from the posterior subcutaneous white AT depot (composed of dorsolumbar, inguinal and gluteal portions), gonadal white AT depot and the interscapular brown AT depot. To obtain the AT stromal vascular fraction (SVF), depots were minced then digested using $1 \mathrm{mg} / \mathrm{mL}$ type II collagenase and cells were passed through a $40 \mu \mathrm{m}$ sieve to remove undigested particles. The same digestion procedure was applied for lung tissue with the addition of $1 \mu \mathrm{g} / \mathrm{mL}$ DNaseI. Spleens were homogenised in cold PBS.
Centrifugation at $500 \times g$ for 10 min was then performed to separate the SVF pellet from floating adipocytes, for downstream usage. AT explants were performed ${ }^{25}$, with supernatants harvested after $2 \mathrm{~h}$ of culture before being frozen and stored for further analysis.

Adipose H\&E staining. Sections of subcut AT were mounted and stained with haematoxylin and eosin according to routine protocols. H\&E sections were imaged on an Aperio XT Slide Scanner and processing was undertaken using Aperio ImageScope software.

Liver biochemical assays. Liver was snap-frozen in liquid nitrogen and stored at $-80^{\circ} \mathrm{C}$ until lipid extraction. Lipids were extracted from powdered tissue using a modified Folch method ${ }^{63}$. The lipid extract was dried under a steady-stream of nitrogen. Extracts were resuspended in $0.4 \mathrm{~mL} 95 \%$ ethanol and heated to $37^{\circ} \mathrm{C}$ prior to lipid assays. Colorimetric assays were used to measure triglycerides (Point Scientific) and cholesterol (Thermo Fisher). All assays were conducted according to the manufacturer's protocols and results were normalised to liver weight.

Cell culture. All cell lines were incubated in a $37^{\circ} \mathrm{C} 5 \% \mathrm{CO}_{2}$ water-jacketed incubator. COS-7 cells were a gift from Stuart Orkin (Harvard), and were cultured in DMEM supplemented with $10 \%$ foetal bovine serum (FBS) and $1 \%$ penicillin-streptomycin-glutamine (PSG). During passaging, adherent cells were lifted after a $5 \mathrm{~min}$ incubation at $37^{\circ} \mathrm{C}$ with $2 \mathrm{mM}$ PBS-EDTA. EoL-1 cells were grown in RPMI 1640 supplemented with 10\% FBS and 1\% PSG. The EoL-1 cell line was supplied by the European Collection of Cell Cultures (ECACC; Salisbury, United Kingdom) as catalogue number 94042252, and purchased from Sigma Aldrich. The cell line has been reported before ${ }^{64}$.

Genome editing. For CRISPR-Cas9 genome editing, a plasmid encoding both the Cas9 protein and the sgRNA was used to delete KLF3 in EoL-1 cells via doublestrand breakage and non-homologous end joining. pSpCas9(BB)-2A-GFP (PX458) was a gift from Feng Zhang (Addgene plasmid 48138) ${ }^{65}$. We designed sgRNA sequences using the Optimized CRISPR-Cas9 design v1 online tool provided by the Zhang laboratory from the Massachusetts Institute of Technology. EoL-1 cells were transfected by nucleofection using a Neon Transfection System (Life Technologies). Cells $\left(5 \times 10^{5}\right)$ were resuspended in nucleofection buffer R (Neon Transfection Kit) and given one pulse of $1350 \mathrm{~V}$ for $30 \mathrm{~ms}$. Cells were then cultured for $72 \mathrm{~h}$ in RPMI 1640 with $10 \%$ FBS but lacking antibiotics. Transfected cells were enriched by FACS, and clonal populations were established by sorting single cells into 96-well culture plates. To screen clones for the desired KLF3 disruption, PCR was carried out on genomic DNA using Q5 polymerase (New England BioLabs), before confirmation via Sanger sequencing of PCR products. sgRNA, PCR and sequencing oligonucleotides can be found in Supplementary Table 2.

Flow cytometry. Flow cytometry was performed using a BD LSRFortessa and BD LSRFortessa X-20. Sorting by FACS was performed using a BD Influx and BD FACS Aria II. All cells were pre-blocked with anti-CD16/32 Fc block to reduce non-specific binding, and UltraComp eBeads (Invitrogen) were used for singlestained compensation controls. For identifying eosinophils, cells were stained with a combination of the following antibodies: CD45-biotin (BD Pharmingen), Streptavidin-BV711 (BD Horizon), CD11b-FITC (BD Pharmingen), F4/80-PE/Cy7 (Biolegend) and SiglecF-BV421 (BD Horizon). Live cells were identified by the addition of TO-PRO-3 viability dye. Eosinophils were defined as live CD45 ${ }^{+}$ $\mathrm{CD}_{11 \mathrm{~b}}{ }^{+} \mathrm{F} 4 / 80^{+}$Siglec-F $\mathrm{F}^{+}$SSChi cells. For delayed flow cytometric analysis, fully stained cells were fixed with $1 \%$ paraformaldehyde. In these instances, ZombieNIR (Biolegend), a fixable viability dye, was used for identifying live cells, and F4/80 was conjugated to PE/Cy5 (eBioscience). To analyse other AT immune cell populations, various fluorescently-conjugated antibodies were used and detailed antibody information can be found in Supplementary Table 3, with gating strategies for immune cell populations available in the legends for Fig. 4 and Supplementary Fig. 8. Flow cytometry analysis was performed using FlowJo software v10.

Co-culture experiments. Culture and differentiation of primary mouse adipocytes was performed ${ }^{66}$. Briefly, isolated SVF cells from the inguinal subcutaneous fat of WT mice were differentiated in 12-well plates for two days using an induction medium of DMEM-F12 GlutaMAX with $10 \%$ FBS and $1 X$ PSG, $1 \mu \mathrm{g} / \mathrm{mL}$ insulin, $0.5 \mathrm{mM}$ IBMX, $0.25 \mu \mathrm{M}$ dexamethasone, $1 \mu \mathrm{M}$ rosiglitazone and $1 \mathrm{nM}$ triiodothyronine (T3). Two days after induction, cells were cultured in maintenance medium (DMEM-F12 GlutaMAX containing 10\% FBS, 1X PSG, $1 \mu \mathrm{M}$ rosiglitazone, $1 \mathrm{nM}$ T3 and $1 \mu \mathrm{g} / \mathrm{mL}$ insulin) which was refreshed every two days. Coculture experiments were performed in a bicompartmental system using $0.4 \mu \mathrm{m}$ pore polycarbonate transwell inserts (Corning). A standardised number of adipose eosinophils were sorted from WT and $\mathrm{Klf3}^{-/-}$subcutaneous SVF using a BD FACS Aria III then resuspended in RPMI containing 10\% FBS, 1 X PSG and $10 \mathrm{ng} / \mathrm{mL}$ IL5. Eosinophils (or media alone) were then added to the upper compartment of the transwell and incubated at $37^{\circ} \mathrm{C}$ in $5 \% \mathrm{CO}_{2}$ for $4 \mathrm{~h}$. Adipocytes from the lower compartment were then collected for gene expression analysis by qPCR. 
Gene expression analysis. To assess mRNA expression, total RNA was isolated from cells and tissues then subjected to cDNA synthesis ${ }^{29}$. Quantitative real-time PCR (qPCR) reactions were set up with Power SYBR Green PCR Master Mix and were run with default cycle parameters on the Applied Biosystems 7500 Fast RealTime PCR System (for 96-well plate format) or the Applied Biosystems ViiA7 RealTime PCR System (for 384-well plate format). Applied Biosystems 7500 software v2.3 and Applied Biosystems QuantStudio Real-Time PCR software v1.3 were used for $\mathrm{qPCR}$ data analysis. Gene expression was quantified using the $2^{-\Delta \Delta \mathrm{CT}}$ method and relative mRNA expression was normalised to $18 \mathrm{~S}$ rRNA levels which have been shown to display consistent expression across the cells and conditions studied. All qPCR primers were designed using primer3 (http://primer3.ut.ee/) and can be found in Supplementary Table 2. For microarrays on eosinophils sorted from subcut SVF by FACS, RNA was isolated using the RNeasy Micro Kit (Qiagen) then subjected to quality control using an Agilent 2100 Bioanalyzer, following preparation with an Agilent RNA 6000 Pico Kit. An Affymetrix 3' IVT Pico Kit was used prior to microarrays which were performed on an Affymetrix GeneChip HT MG-430PM Array Plate. Partek Genomics Suite v7 software was used for data analysis, and microarray datasets are available from GEO (Accession No. GSE117445).

Protein extraction and quantification. Whole-cell protein extracts (WCE) were prepared by homogenising mouse tissues with a glass dounce, in the presence of radioimmunoprecipitation (RIPA) buffer (50 mM HEPES, pH 7.5; $500 \mathrm{mM} \mathrm{LiCl;} 1$ mM EDTA; $1 \%$ NP-40; $0.7 \%$ sodium deoxycholate) containing protease inhibitors (cOmplete, Mini, EDTA-free Protease Inhibitor Cocktail; one tablet dissolved in 10 $\mathrm{mL}$ RIPA buffer). Homogenates were rotated at $4^{\circ} \mathrm{C}$ for $1 \mathrm{~h}$ then centrifuged at $21,000 \times g$ for $20 \mathrm{~min}$ at $4^{\circ} \mathrm{C}$ to obtain the WCE. Protein extracts were subjected to a bicinchoninic acid (BCA) assay using a BCA Protein Assay Kit (Pierce) to determine concentration according to the manufacturer's protocol. For detection of UCP1, $25 \mu \mathrm{g}$ of WCE was loaded onto Novex NuPAGE $10 \%$ Bis-Tris gels, following denaturation and boiling ( $15 \mu \mathrm{g}$ for brown AT WCE). After blocking, nitrocellulose membranes were incubated overnight with anti-UCP1 antibody (Abcam). Membranes were then probed with HRP-linked anti-rabbit antibody (GE Healthcare) prior to exposure using the GE ImageQuant LAS 500 in the presence of Immobilon Western Chemiluminescent HRP Substrate (Millipore). To detect expression of VDAC (voltage-dependent anion channel), $20 \mu \mathrm{g}$ of WCE was used and incubation took place overnight with anti-VDAC antibody (Cell Signaling) before probing with HRP-linked anti-rabbit secondary antibody. To detect mitochondrial electron transport chain complexes, several deviations from the above protocol were performed. Briefly, WCE were boiled for $5 \mathrm{~min}$ at $50^{\circ} \mathrm{C}$ before SDSpolyacrylamide gel electrophoresis (PAGE) and transferral to a $0.45 \mu \mathrm{m}$ polyvinyl difluoride (PVDF) membrane. Blocking took place overnight at $4{ }^{\circ} \mathrm{C}$ using $5 \%(\mathrm{w} /$ v) skim milk powder in PBS (pH 7.4) with gentle agitation, followed by washing in PBST (PBS with $0.1 \%$ Tween-20). The membrane was incubated with the primary Total OXPHOS Rodent WB Antibody Cocktail (Abcam) in 1\% skim (w/v)/PBS. This optimised primary antibody mix contains five mouse antibodies, one each against the mitochondrial oxidative phosphorylation (oxphos) complexes (Complex I: NADH dehydrogenase; Complex II: succinate dehydrogenase; Complex III: $\mathrm{CoQH}_{2}$-cytochrome $c$ reductase; Complex IV: cytochrome $c$ oxidase; Complex V: ATP synthase). The secondary HRP-linked anti-mouse antibody labelling was performed in $1 \%$ skim milk (w/v)/PBS before imaging. All membranes were stripped with $0.5 \mathrm{M} \mathrm{NaOH}$ then re-blocked with skim milk before probing with an anti- $\beta$-actin antibody (Sigma). Following this, HRP-linked anti-mouse antibody (GE Healthcare) was incubated on membranes prior to imaging. For measurement of meteorin-like and IL-33 levels in plasma and AT explant supernatant, mouse meteorin-like/METRNL DuoSet and mouse IL-33 DuoSet ELISA kits were utilised according to manufacturer's instructions (R\&D Systems). Detailed antibody information can be found in Supplementary Table 3.

Chromatin immunoprecipitation. ChIP experiments were performed ${ }^{67}$. Briefly, $\sim 7 \times 10^{7}$ cells were used for each immunoprecipitation (IP). Cells were crosslinked with $1 \%$ formaldehyde for $10 \mathrm{~min}$ at room temperature, and the reaction was quenched with glycine at a final concentration of $125 \mathrm{mM}$. Crosslinked cells were then lysed and sonicated to obtain $\sim 200-400$ bp fragments of chromatin. Crosslinked DNA was pulled down at $4{ }^{\circ} \mathrm{C}$ overnight using $15 \mu \mathrm{g}$ anti-KLF3 antibody (Thermo Fisher) or $15 \mu \mathrm{g}$ control normal goat IgG (Santa Cruz Biotechnology) per IP. qPCR was performed on ChIP material using an Applied Biosystems ViiA7 Real-Time PCR System. For analysis, IPs were first normalised to the relative amount of input DNA, then to IgG controls. All qPCR primers for ChIP were designed using primer3 (http://primer3.ut.ee/) and can be found in Supplementary Table 2. A ChIP-Seq data set for V5-tagged KLF3 produced from murine embryonic fibroblasts was obtained from GEO (Accession No. GSE44748) ${ }^{52}$ and used to assess genome-wide KLF3 binding using Integrative Genomics Viewer (Broad Institute) ${ }^{68,69}$. Detailed antibody information can be found in Supplementary Table 3.

Software. GraphPad Prism v8 was used for generation of graphs and statistical analysis. Adobe Photoshop and Illustrator CS5.1 were used for compiling and creating figures. FlowJo v10 was used for flow cytometry and cell sorting analysis.
Applied Biosystems 7500 v2.3 and QuantStudio Real-Time PCR v1.3 were used for running and analysing $\mathrm{QPCR}$ experiments. Oligonucleotides were designed using the online primer3 tool v4.1.0, and the Optimized CRISPR design v1 online tool was used for designing sgRNAs. Integrative Genomics Viewer v2.4.9 (Broad Institute) was used for viewing ChIP-Seq data. Bio-Rad Image Lab v6.0.1 software was used for capturing and analysing agarose gels. Aperio ImageScope software was used for analysing H\&E sections. Licensed BioRender.com online software was used for generating cartoons and figures.

Statistical analysis. The mean is shown for data in each figure with individual data points shown, and SEM is shown as error bars. For flow cytometry plots, the median representative plot for each genotype/condition is shown, accompanied by the means \pm SEM. D'Agostino-Pearson normality tests were performed to determine whether data followed a Gaussian distribution. Given the sample sizes, nonnormality was determined, and thus non-parametric one-tailed Mann-Whitney $U$ tests were used to test specific, directional hypotheses. ${ }^{*} P<0.05,{ }^{* *} P<0.01$, ${ }^{* * *} P$ $<0.001$ (or ${ }^{\#} P<0.05$ where stated). Two-way ANOVA followed by post-hoc Tukey testing was carried out to assess significance between genotype, body temperature and time in acute cold and thermoneutral experiments. Two-way ANOVA followed by False Discovery Rate to test for multiple comparisons was used for significant differentially-expressed genes in microarray studies. Fisher's Exact test was used to define significantly enriched GO terms in microarray Forest Plots. All statistical analyses were performed in GraphPad Prism v8.

Reporting summary. Further information on research design is available in the Nature Research Reporting Summary linked to this article.

\section{Data availability}

Data supporting the findings of this study can be found within the paper and its Supplementary Information files, and a Source data file has been provided for data underlying figures. Further information is available upon reasonable request. Full scans of western blots are available in Supplementary Fig. 10. Datasets are publically available from the NCBI Gene Expression Omnibus (GEO) using Accession Nos. GSE117445 and GSE44748, FANTOM5 SSTAR https://fantom.gsc.riken.jp/5/sstar/Main_Page) and Haemosphere (haemosphere.org/). A reporting summary has also been provided as part of the Supplementary Information files. Source data are provided with this paper.

Received: 7 November 2018; Accepted: 20 May 2020;

Published online: 10 June 2020

\section{References}

1. $\mathrm{Wu}, \mathrm{J}$. et al. Beige adipocytes are a distinct type of thermogenic fat cell in mouse and human. Cell 150, 366-376 (2012).

2. Wu, J., Cohen, P. \& Spiegelman, B. M. Adaptive thermogenesis in adipocytes: is beige the new brown? Genes Dev. 27, 234-250 (2013).

3. Qiu, Y. et al. Eosinophils and type 2 cytokine signaling in macrophages orchestrate development of functional beige fat. Cell 157, 1292-1308 (2014).

4. Wu, D. et al. Eosinophils sustain adipose alternatively activated macrophages associated with glucose homeostasis. Science 332, 243-247 (2011).

5. Rao, R. R. et al. Meteorin-like is a hormone that regulates immune-adipose interactions to increase beige fat thermogenesis. Cell 157, 1279-1291 (2014).

6. Lee, E.-H. et al. Eosinophils support adipocyte maturation and promote glucose tolerance in obesity. Sci. Rep. 8, 9894 (2018).

7. Bolus, W. R. et al. Elevating adipose eosinophils in obese mice to physiologically normal levels does not rescue metabolic impairments. Mol. Metab. 8, 86-95 (2018).

8. Nguyen, K. D. et al. Alternatively activated macrophages produce catecholamines to sustain adaptive thermogenesis. Nature 480, 104-108 (2011).

9. Pirzgalska, R. M. et al. Sympathetic neuron ${ }^{-}$associated macrophages contribute to obesity by importing and metabolizing norepinephrine. Nat. Med 23, 1309-1318 (2017).

10. Camell, C. D. et al. Inflammasome-driven catecholamine catabolism in macrophages blunts lipolysis during ageing. Nature 550, 119-123 (2017).

11. Fischer, K. et al. Alternatively activated macrophages do not synthesize catecholamines or contribute to adipose tissue adaptive thermogenesis. Nat. Med 23, 623-630 (2017).

12. Bell-Anderson, K. S. et al. Loss of Kruppel-like factor 3 (KLF3/BKLF) leads to upregulation of the insulin-sensitizing factor adipolin (FAM132A/CTRP12/ C1qdc2). Diabetes 62, 2728-2737 (2013).

13. Sue, N. et al. Targeted disruption of the basic Kruppel-like factor gene (Klf3) reveals a role in adipogenesis. Mol. Cell Biol. 28, 3967-3978 (2008).

14. Brestoff, J. R. et al. Group 2 innate lymphoid cells promote beiging of white adipose tissue and limit obesity. Nature 519, 242-246 (2015). 
15. Lee, M. W. et al. Activated type 2 innate lymphoid cells regulate beige fat biogenesis. Cell 160, 74-87 (2015).

16. Min, S. Y. et al. Human 'brite/beige' adipocytes develop from capillary networks, and their implantation improves metabolic homeostasis in mice. Nat. Med. 22, 312-318 (2016).

17. Jia, R. et al. Characterization of cold-induced remodelling reveals depotspecific differences across and within brown and white adipose tissues in mice. Acta Physiol. 217, 311-324 (2016).

18. Ghorbani, M. \& Himms-Hagen, J. Appearance of brown adipocytes in white adipose tissue during CL 316,243-induced reversal of obesity and diabetes in Zucker fa/fa rats. Int. J. Obes. (Lond.) 21, 465-475 (1997).

19. Harms, M. \& Seale, P. Brown and beige fat: development, function and therapeutic potential. Nat. Med. 19, 1252-1263 (2013).

20. Seale, P. et al. Prdm16 determines the thermogenic program of subcutaneous white adipose tissue in mice. J. Clin. Investig. 121, 96-105 (2011).

21. Roh, H. C. et al. Warming Induces Significant Reprogramming of Beige, but Not Brown, Adipocyte Cellular Identity. Cell Metab. 27, 1121-1137. e5 (2018).

22. Pollard, A. E. et al. AMPK activation protects against diet-induced obesity through Ucp1-independent thermogenesis in subcutaneous white adipose tissue. Nat. Metab. 1, 340-349 (2019).

23. Ikeda, K. et al. UCP1-independent signaling involving SERCA2b-mediated calcium cycling regulates beige fat thermogenesis and systemic glucose homeostasis. Nat. Med. 23, 1454-1465 (2017).

24. Kazak, L. et al. Ablation of adipocyte creatine transport impairs thermogenesis and causes diet-induced obesity. Nat. Metab. 1, 360-370 (2019).

25. Kazak, L. et al. A creatine-driven substrate cycle enhances energy expenditure and thermogenesis in beige fat. Cell 163, 643-655 (2015).

26. Ye, L. et al. Fat cells directly sense temperature to activate thermogenesis. Proc. Natl Acad. Sci. USA 110, 12480-12485 (2013).

27. Davis, T. R. Chamber cold acclimatization in man. J. Appl. Physiol. 16, 1011-1015 (1961).

28. Passmore, R. \& Durnin, J. V. Human energy expenditure. Physiol. Rev. 35, 801-840 (1955).

29. Knights, A. J. et al. Kruppel-like factor 3 (KLF3/BKLF) is required for widespread repression of the inflammatory modulator galectin-3 (Lgals3). J. Biol. Chem. 291, 16048-16058 (2016).

30. Knights, A. J. et al. Krüppel-like factor 3 (KLF3) suppresses NF-kB-driven inflammation in mice. J. Biol. Chem. https://doi.org/10.1074/jbc. RA120.013114 (2020).

31. Funnell, A. P. et al. Generation of mice deficient in both KLF3/BKLF and KLF8 reveals a genetic interaction and a role for these factors in embryonic globin gene silencing. Mol. Cell Biol. 33, 2976-2987 (2013).

32. Funnell, A. P. et al. The CACCC-binding protein KLF3/BKLF represses a subset of KLF1/EKLF target genes and is required for proper erythroid maturation in vivo. Mol. Cell Biol. 32, 3281-3292 (2012).

33. $\mathrm{Vu}, \mathrm{T}$. T. et al. Impaired B cell development in the absence of Kruppel-like factor 3. J. Immunol. 187, 5032-5042 (2011).

34. Heng, T. S. \& Painter, M. W. The Immunological Genome Project: networks of gene expression in immune cells. Nat. Immunol. 9, 1091-1094 (2008).

35. Lucas, E. C. et al. Haemopedia RNA-seq: a database of gene expression during haematopoiesis in mice and humans. Nucleic Acids Res. 47, D780-D785 (2018).

36. de Graaf, C. A. et al. Haemopedia: An Expression Atlas of Murine Hematopoietic Cells. Stem Cell Rep. 7, 571-582 (2016).

37. Abugessaisa, I. et al. FANTOM5 transcriptome catalog of cellular states based on Semantic MediaWiki. Database 2016, baw105 (2016).

38. Bouffi, C. et al. Transcription factor repertoire of homeostatic eosinophilopoiesis. J. Immunol. 195, 2683-2695 (2015).

39. Lee, S. D. \& Tontonoz, P. Eosinophils in fat: pink is the new brown. Cell 157, 1249-1250 (2014).

40. Knights, A. J., Vohralik, E. J., Hoehn, K. L., Crossley, M. \& Quinlan, K. G. R. Defining eosinophil function in adiposity and weight loss. Bioessays $\mathbf{4 0}$, e1800098 (2018).

41. Vohralik, E. J., Psaila, A. M., Knights, A. J. \& Quinlan, K. G. R. EoTHINophils: eosinophils as key players in adipose tissue homeostasis. Clin. Exp. Pharmacol. Physiol. https://doi.org/10.1111/1440-1681.13304 (2020).

42. Eaton, S. A. et al. A network of Kruppel-like factors (Klfs). Klf8 is repressed by Klf3 and activated by Klf1 in vivo. J. Biol. Chem. 283, 26937-26947 (2008).

43. Suo, J., Zhao, X., Guo, X. \& Zhao, X. Met-enkephalin improves metabolic syndrome in high fat diet challenged mice through promotion of adipose tissue browning. Toxicol. Appl. Pharm. 359, 12-23 (2018).

44. Zuberi, R. I. et al. Critical role for galectin-3 in airway inflammation and bronchial hyperresponsiveness in a murine model of asthma. Am. J. Pathol. 165, 2045-2053 (2004)

45. Ge, X. N., Ha, S. G., Liu, F. T., Rao, S. P. \& Sriramarao, P. Eosinophilexpressed galectin-3 regulates cell trafficking and migration. Front Pharm. 4, 37 (2013).
46. Vasanthakumar, A. et al. The transcriptional regulators IRF4, BATF and IL-33 orchestrate development and maintenance of adipose tissue-resident regulatory T cells. Nat. Immunol. 16, 276-285 (2015).

47. Han, J. M. et al. IL-33 Reverses an obesity-induced deficit in visceral adipose tissue ST2+ T regulatory cells and ameliorates adipose tissue inflammation and insulin resistance. J. Immunol. 194, 4777-4783 (2015).

48. Spallanzani, R. G. et al. Distinct immunocyte-promoting and adipocytegenerating stromal components coordinate adipose tissue immune and metabolic tenors. Sci Immunol. 4, eaaw3658 (2019).

49. Mahlakoiv, T. et al. Stromal cells maintain immune cell homeostasis in adipose tissue via production of interleukin-33. Sci. Immunol. 4, eaax0416 (2019).

50. Moysidou, M. et al. CD8 $+\mathrm{T}$ cells in beige adipogenesis and energy homeostasis. JCI Insight 3, e95456 (2018).

51. Rajbhandari, P. et al. IL-10 signaling remodels adipose chromatin architecture to limit thermogenesis and energy expenditure. Cell 172, 218-233. e217 (2018).

52. Burdach, J. et al. Regions outside the DNA-binding domain are critical for proper in vivo specificity of an archetypal zinc finger transcription factor. Nucleic Acids Res. 42, 276-289 (2014).

53. Coats, B. R. et al. Metabolically activated adipose tissue macrophages perform detrimental and beneficial functions during diet-induced obesity. Cell Rep. 20, 3149-3161 (2017)

54. Lumeng, C. N., Bodzin, J. L. \& Saltiel, A. R. Obesity induces a phenotypic switch in adipose tissue macrophage polarization. J. Clin. Investig. 117, 175-184 (2007).

55. Jun, $\mathrm{H}$. et al. An immune-beige adipocyte communication via nicotinic acetylcholine receptor signaling. Nat. Med. 24, 814-822 (2018).

56. Fabbiano, S. et al. Caloric restriction leads to browning of white adipose tissue through type 2 immune signaling. Cell Metab. 24, 434-446 (2016).

57. Abdullahi, A. et al. IL-6 signal from the bone marrow is required for the browning of white adipose tissue post burn injury. Shock 47, 33-39 (2017).

58. Haldar, S. M. et al. Kruppel-like factor 15 regulates skeletal muscle lipid flux and exercise adaptation. Proc. Natl Acad. Sci. USA 109, 6739-6744 (2012).

59. Liao, X. et al. Kruppel-like factor 4 is critical for transcriptional control of cardiac mitochondrial homeostasis. J. Clin. Investig. 125, 3461-3476 (2015).

60. Oishi, Y. et al. SUMOylation of Kruppel-like transcription factor 5 acts as a molecular switch in transcriptional programs of lipid metabolism involving PPAR-delta. Nat. Med 14, 656-666 (2008).

61. Loft, A. et al. Browning of human adipocytes requires KLF11 and reprogramming of PPARgamma superenhancers. Genes Dev. 29, 7-22 (2015).

62. Guillaumond, F. et al. Kruppel-like factor KLF10 is a link between the circadian clock and metabolism in liver. Mol. Cell Biol. 30, 3059-3070 (2010).

63. Folch, J., Lees, M. \& Sloane Stanley, G. H. A simple method for the isolation and purification of total lipides from animal tissues. J. Biol. Chem. 226, 497-509 (1957).

64. Saito, H. et al. Establishment and characterization of a new human eosinophilic leukemia cell line. Blood 66, 1233-1240 (1985).

65. Ran, F. A. et al. Genome engineering using the CRISPR-Cas9 system. Nat. Protoc. 8, 2281-2308 (2013).

66. Chung, K.-J. et al. A self-sustained loop of inflammation-driven inhibition of beige adipogenesis in obesity. Nat. Immunol. 18, 654-664 (2017).

67. Schmidt, D. et al. ChIP-seq: using high-throughput sequencing to discover protein-DNA interactions. Methods 48, 240-248 (2009).

68. Robinson, J. T. et al. Integrative genomics viewer. Nat. Biotechnol. 29, 24-26 (2011).

69. Thorvaldsdottir, H., Robinson, J. T. \& Mesirov, J. P. Integrative Genomics Viewer (IGV): high-performance genomics data visualization and exploration. Brief. Bioinform. 14, 178-192 (2013).

\section{Acknowledgements}

We would like to thank Chris Brownlee and Emma Johansson at the UNSW BRIL Flow Cytometry Facility for their assistance with flow cytometry and sorting experiments, along with Brendan Lee (UNSW BRIL) and Debbie Burnett (Garvan Institute) for their help with animal experiments. We would like to acknowledge the work of the Garvan Histopathology Service for histological sample preparation and staining, and the Biomedical Imaging Facility (BMIF) at UNSW Sydney. We are also very grateful for the assistance of Dr. Axel Kallies, Dr. Ajith Vasanthakumar and Dr. Jon Brestoff with analysing immune cell populations by flow cytometry. We would also like to acknowledge the assistance of the Ramaciotti Centre for Genomics (UNSW) for their sequencing and microarray assistance. This work was supported by funding from the Australian National Health and Medical Research Council to M.C. (APP1025873 and APP1025877). A.J.K., J.J.Y. and E.S.S. were supported by Australian Postgraduate Awards. H.M.J. was supported by an Academic Training Scheme (SLAB) scholarship from the Malaysian Ministry of Higher Education. K.G.R.Q. was supported by a Scientia Fellowship and S.J. A. and E.J.V. were supported by Scientia Scholarships. 


\section{Author contributions}

A.J.K., K.G.R.Q. and M.C. wrote the paper. A.J.K. performed most of the experiments; E.J.V., P.J.H. and A.J.K. performed the acute cold and thermoneutral experiments under the supervision of K.G.R.Q. and K.N.N.; S.J.A. performed liver biochemical assays; J.J.Y. generated the KLF3 CRISPR construct; H.M.J. and K.S.B.A. performed the AT explant experiments; E.S. S., L.J.N. and E.M.O. performed preliminary studies and provided technical assistance with experiments. A.J.K., K.G.R.Q. and M.C. designed the study, with intellectual input provided by K.L.H. All authors reviewed the results and approved the final version of the paper.

\section{Competing interests}

The authors declare no competing interests.

\section{Additional information}

Supplementary information is available for this paper at https://doi.org/10.1038/s41467020-16758-9.

Correspondence and requests for materials should be addressed to K.G.R.Q.

Peer review information Nature Communications thanks Konstantinos Drosatos and the other, anonymous, reviewer(s) for their contribution to the peer review of this work.
Reprints and permission information is available at http://www.nature.com/reprints

Publisher's note Springer Nature remains neutral with regard to jurisdictional claims in published maps and institutional affiliations.

\section{(c) (i)}

Open Access This article is licensed under a Creative Common Attribution 4.0 International License, which permits use, sharing, adaptation, distribution and reproduction in any medium or format, as long as you give appropriate credit to the original author(s) and the source, provide a link to the Creative Commons license, and indicate if changes were made. The images or other third party material in this article are included in the article's Creative Commons license, unless indicated otherwise in a credit line to the material. If material is not included in the article's Creative Commons license and your intended use is not permitted by statutory regulation or exceeds the permitted use, you will need to obtain permission directly from the copyright holder. To view a copy of this license, visit http://creativecommons.org/ licenses/by/4.0/.

(c) The Author(s) 2020 